\section{(B) OPEN ACCESS}

\title{
Prescription of secondary prevention medications, lifestyle advice, and referral to rehabilitation among acute coronary syndrome inpatients: results from a large prospective audit in Australia and New Zealand
}

\author{
Julie Redfern, ${ }^{1,2}$ Karice Hyun, ${ }^{1,2}$ Derek $\mathrm{P}$ Chew $^{3}{ }^{3}$ Carolyn Astley, ${ }^{4}$ Clara Chow, ${ }^{1,2,5}$ \\ Bernadette Aliprandi-Costa, ${ }^{6}$ Tegwen Howell, ${ }^{1,7}$ Bridie Carr, ${ }^{8}$ Karen Lintern, ${ }^{8}$ \\ Isuru Ranasinghe, ${ }^{6}$ Kellie Nallaiah, ${ }^{1}$ Fiona Turnbull, ${ }^{1,2}$ Cate Ferry, ${ }^{9}$ Chris Hammett, ${ }^{10}$ \\ Chris J Ellis, ${ }^{11}$ John French, ${ }^{12,13}$ David Brieger, ${ }^{2,6}$ Tom Briffa $^{14}$
}

For numbered affiliations see end of article.

\section{Correspondence to Associate Professor Julie Redfern, Cardiovascular Division, The George Institute for Global Health, Level 10, King George V Building, Missenden Road, Camperdown, NSW 2050, Australia; jredfern@ georgeinstitute.org.au}

Received 21 November 2013 Revised 23 April 2014 Accepted 24 April 2014 Published Online First 9 June 2014

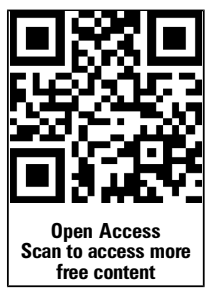

\section{SLinked}

- http://dx.doi.org/10.1136/ heartjnl-2014-306114

\section{CrossMark}

To cite: Redfern J, Hyun K, Chew DP, et al. Heart 2014; 100:1281-1288

\section{ABSTRACT}

Objective To evaluate the proportion of patients hospitalised with acute coronary syndrome (ACS) in Australia and New Zealand who received optimal inpatient preventive care and to identify factors associated with preventive care.

Methods All patients hospitalised bi-nationally with ACS were identified between 14-27 May 2012. Optimal in-hospital preventive care was defined as having received lifestyle advice, referral to rehabilitation, and prescription of secondary prevention pharmacotherapies. Multilevel multivariable logistic regression was used to determine factors associated with receipt of optimal preventive care.

Results For the 2299 ACS survivors, mean (SD) age was 69 (13) years, $46 \%$ were referred to rehabilitation, $65 \%$ were discharged on sufficient preventive medications, and 27\% received optimal preventive care. Diagnosis of ST elevation myocardial infarction (OR: 2.64 [95\% Cl: 1.88-3.71]; $p<0.001)$ and non-ST elevation myocardial infarction (OR: 1.99 [95\% Cl: 1.52-2.61]; $p<0.001)$ compared with a diagnosis of unstable angina, having a percutaneous coronary intervention (PCI) (OR: 4.71 [95\% Cl: 3.67-6.11]; $p<0.001$ ) or coronary bypass (OR: 2.10 [95\% Cl: 1.21-3.60]; $p=0.011)$ during the admission or history of hypertension (OR:1.36 [95\% Cl: 1.06-1.75]; $p=0.017$ ) were associated with greater exposure to preventive care. Age over 70 years (OR:0.53 [95\% Cl: $0.35-0.79]$; $\mathrm{p}=0.002$ ) or admission to a private hospital (OR:0.59 [95\% Cl: 0.42-0.84]; $p=0.003$ ) were associated with lower exposure to preventive care.

Conclusions Only one-quarter of ACS patients received optimal secondary prevention in-hospital. Patients with $\mathrm{UA}$, who did not have $\mathrm{PCl}$, were over 70 years or were admitted to a private hospital, were less likely to receive optimal care.

\section{BACKGROUND}

Cardiovascular disease (CVD), including coronary heart disease (CHD) and stroke, is the leading cause of death and disease burden globally. ${ }^{1}$ In Australia, CHD accounts for the greatest disease morbidity and nearly one fifth of all deaths nationally. $^{2}$ The potentially life-threatening presentation with CHD is a spectrum of clinical conditions known as acute coronary syndrome (ACS) which includes ST segment elevation myocardial infarction (STEMI), non-ST segment elevation MI (NSTEMI), and unstable angina (UA). ${ }^{3}$ Importantly, approximately half of these events occur in individuals who have had a prior hospital admission for CHD. ${ }^{3-5}$ European, ${ }^{6} 7$ American, ${ }^{8}$ and Australian ${ }^{3}$ guidelines routinely recommend strategies aimed at preventive care, preferably commencing while the patient is in hospital. These guidelines emphasise the importance of secondary prevention pharmacotherapy, lifestyle advice, and participation in a cardiac rehabilitation or secondary prevention programme.

Cross-sectional surveys and registries are a valuable means for assessing the implementation of guidelines. ${ }^{9-11}$ Indeed, international registries have demonstrated suboptimal pharmacotherapy and referral to cardiac rehabilitation at discharge across various settings. ${ }^{9-11}$ The first Euro Heart Survey of ACS, conducted in 25 countries in Europe and the Mediterranean basin in 2000-2001, demonstrated substantial variability in the implementation of guidelines applicable at that time. ${ }^{12}$ A second Euro Heart Survey in 2004 among 32 countries, which aimed to reassess ACS management and implementation of more contemporary guidelines, showed persistent gaps in chronic care. ${ }^{10}$ New Zealand researchers have also demonstrated low levels of investigations, appropriate pharmacotherapy treatments, and acute revascularisation in previous audits of ACS care in 2002 and 2007. ${ }^{13} 14$

ACS registries have provided valuable information about treatment and resource gaps ${ }^{15}$; however, they frequently include relatively few patients from regional and remote centres. ${ }^{4}$ In addition, very few registries have reported on the synergistic impact of comprehensive care comprising medications, lifestyle advice, and post-discharge preventive activities. This is particularly important given there is evidence that medications started in hospital are 
more likely to be continued ${ }^{16}$ and recent findings that early referral to cardiac rehabilitation improves later attendance at programme orientation. ${ }^{17}$ The inclusion of all consenting acute medical services in Australia and New Zealand providing immediate care to patients presenting with suspected ACS allows a unique opportunity to collect a complete perspective on patterns of ACS care across both countries. This paper evaluates the proportion of patients admitted to an Australian or New Zealand hospital with ACS, surviving to discharge, who received optimal in-hospital preventive care (comprising medications, lifestyle advice, and referral to rehabilitation). We also aimed to identify important clinical factors that were associated with exposure to optimal preventive care during an ACS admission.

\section{METHODS}

\section{Study design}

The SNAPSHOT ACS study was a prospective audit of the care provided to consecutive patients admitted to an Australian or New Zealand hospital with suspected ACS during a specified 2 -week period. ${ }^{15}$ The study was designed by a bi-national academic network of clinicians and researchers and was managed by a steering committee with key stakeholder representation. An overview of the findings is published elsewhere. ${ }^{15}$ This present article specifically focuses on preventive care at the point of discharge and provides a detailed analysis only of those patients who survived their index admission and had a confirmed diagnosis of STEMI, NSTEMI or UA. Written study protocols were provided to all sites and relevant research staff were given training and support for data collection and entry. Ethical approval (with opt-out consent) was obtained for Australian sites and single review (with consent waiver) in New Zealand's case was obtained from all participating sites.

\section{Sites and participants}

All hospitals receiving patients with suspected ACS (including public or private, metropolitan or rural) were identified from public records and invited to participate. Patients were eligible if they were admitted with a suspected or confirmed ACS event between 14 May 00:00 $\mathrm{h}$ and 27 May 24:00 h, 2012. ${ }^{15}$ Consecutive admissions were enrolled and patients were followed for the duration of the acute care episode, including all contiguous transfers between hospitals (counted as a single episode of care).

\section{Data collection}

A common case report form, with standardised completion note, was developed by opinion leaders in collaboration with a multidisciplinary team comprising allied health professionals, biostatisticians, epidemiologists, and data management consultants and trialled across multiple jurisdictions in approximately 60 patients. Data collection was from medical records and focused on presenting characteristics, including clinical variables enabling the calculation of the Global Registry of Acute Coronary Events (GRACE) risk score, ${ }^{18}$ as well as details of patient presentation and transfers between hospitals. Data missing from medical records was record as 'no'. Utilisation of guideline recommended therapies including inpatient invasive management/revascularisation, dietary/physical activity advice, and prior-to-discharge prescription of aspirin, other oral antiplatelet therapies, statin, $\beta$-blocker, ACE inhibitor (ACEi) or angiotensin receptor blocker (ARB), and referral for cardiac rehabilitation were all assessed. Smoking cessation advice was reported separately. Data were collected using a customised database permitting secure, web-based entry of each patient.

\section{Exposure to preventive care}

To quantify exposure to preventive care we devised a composite outcome based on a series of standard variables derived from international guidelines. ${ }^{36-8}$ In combination these guidelines identify three important areas for ongoing preventive care, namely pharmacotherapy, lifestyle advice/change, and cardiac rehabilitation. It is for this reason that we defined optimal preventive care as the patient having received at least one aspect of lifestyle advice in-hospital (exercise or diet advice and quit advice for smokers), referral to cardiac rehabilitation post-discharge, and prescription of at least four of the five major preventive pharmacotherapies (lipid lowering, aspirin, other antiplatelet, $\beta$-blocker, or ACEi/ ARB). This was slightly different for patients with UA and a GRACE risk score $<130$, where prescription of at least two preventive pharmacotherapies (namely aspirin and statin) was used to quantify exposure to preventive care. ${ }^{7}$ Given that the relative contributions of medication, referral to rehabilitation, and lifestyle advice may not be equivalent in terms of outcomes, we also conducted an additional analysis with extra weighting for medications. For this analysis, $50 \%$ weighting was assigned to pharmacotherapy and $50 \%$ weighting was assigned to rehabilitation/risk factor advice, as is consistent with evidence for their relative contribution in reducing $\mathrm{CHD}$ mortality. ${ }^{19} 20$

\section{Statistical analysis}

Patient characteristics, rates of referral to cardiac rehabilitation, provision of guideline recommended medications, and access to inpatient preventive care are presented using standard descriptive statistics stratified by discharge diagnosis. Categorical variables are reported as numbers and percentages (compared using $\chi^{2}$ test) and continuous variables as means and SD or medians and IQRs. We used multilevel multivariable logistic regression models (Proc Glimmix) in all analyses to derive the ORs and corresponding 95\% CIs, where the effect of clustering by hospital was modelled as a random intercept. We identified input variables that were likely to be of clinical importance; these were: age, gender, discharge diagnosis (STEMI, NSTEMI, UA), current smoker, percutaneous coronary intervention (PCI) during index admission, coronary artery bypass graft surgery (CABG) during index admission, prior $\mathrm{PCI}$ or $\mathrm{CABG}$, prior vascular disease (including prior myocardial infarction, peripheral arterial disease, transient ischaemic attack or stroke), history of diabetes mellitus, history of hypertension, history of morbid obesity, and length of index ACS stay (days). Public versus private hospital was the second level predictor variable. In all models, covariates were mutually adjusted and analyses were undertaken using Statistical Analysis Software (SAS) Enterprise Guide 4.3. A value of $p<0.05$ was considered statistically significant for all analyses.

\section{RESULTS}

The main SNAPSHOT ACS results, including state and population data, have been reported previously. ${ }^{14}$ In summary, 4398 patients with suspected or confirmed ACS were identified from a potential pool of 525 hospitals across Australia and New Zealand. ${ }^{14}$ Some 483 hospitals $(92 \%$ of all potential hospitals) gained ethics approval, 444 in Australia (with opt-out consent) and 39 in New Zealand (national consent). In total, 286 sites contributed to recruitment with the 197 non-enrolling hospitals being smaller, outside larger population centres, and not treating patients with suspected ACS during the 2 -week audit window. ${ }^{14}$ Every effort was made to ensure recruitment into the study was maximised with an opt-out consent for all but two hospitals (only 44 potential participants chose to opt-out) and through daily cross-checking 
Table 1 Demographic, clinical and discharge care

\begin{tabular}{|c|c|c|c|c|}
\hline & STEMI $(n=390)$ & NSTEMI $(n=981)$ & UA+chest pain of likely ischaemic origin $(n=928)$ & All ACS $(n=2299)$ \\
\hline \multicolumn{5}{|c|}{ Demographics and documented medical history before index admission* } \\
\hline Mean age, years (SD) & $65(14)$ & $71(13)$ & $68(13)$ & $69(13)$ \\
\hline Male & $283(73)$ & $619(63)$ & $586(63)$ & $1488(65)$ \\
\hline Prior Ml & $64(16)$ & $330(34)$ & $334(36)$ & $728(32)$ \\
\hline Prior $\mathrm{PCl}$ & $44(11)$ & $180(18)$ & $308(33)$ & $532(23)$ \\
\hline Prior $\mathrm{CABG}$ & $16(4)$ & $129(13)$ & $133(14)$ & $278(12)$ \\
\hline History of hypertension & $209(54)$ & $673(69)$ & $676(73)$ & $1558(68)$ \\
\hline History of hyperlipidaemia & $176(45)$ & $571(58)$ & $617(66)$ & $1364(59)$ \\
\hline Diabetes & $69(18)$ & $303(31)$ & $288(31)$ & $660(29)$ \\
\hline Prior PAD or TIA or stroke & $31(8)$ & $197(20)$ & $149(16)$ & $377(16)$ \\
\hline Morbid obesity $\left(\geq 35 \mathrm{~kg} / \mathrm{m}^{2}\right)$ & $21(5)$ & $81(8)$ & $77(8)$ & $179(8)$ \\
\hline Other comorbidity $\dagger$ & $40(10)$ & $219(22)$ & $155(17)$ & $414(18)$ \\
\hline Length of stay for index admission, median days (IQR) & $4.1(2.9,6.5)$ & $4.2(2.9,6.8)$ & $2.1(1.1,4.1)$ & $3.5(2.0,5.8)$ \\
\hline \multicolumn{5}{|c|}{ Documented clinical measures during index admission* } \\
\hline Total cholesterol, mean (SD) & $4.78(1.2)$ & $4.68(1.4)$ & $4.57(1.3)$ & $4.67(1.3)$ \\
\hline$\geq 4.5 \mathrm{mmol} / \mathrm{L}$ & 179/306 (58) & $317 / 605(52)$ & $215 / 438(49)$ & $711 / 1349(53)$ \\
\hline LDL cholesterol, mean (SD) & $2.89(1.0)$ & $2.74(1.1)$ & $2.51(1.2)$ & $2.7(1.1)$ \\
\hline$\geq 2.5 \mathrm{mmol} / \mathrm{L}$ & $174 / 265(66)$ & $290 / 519(56)$ & $155 / 326(48)$ & $619 / 1110(56)$ \\
\hline Glucose, mean (SD) & $8.55(5.5)$ & $9.40(12.8)$ & $8.11(8.8)$ & $8.74(10.3)$ \\
\hline$\geq 6.5 \mathrm{mmol} / \mathrm{L}$ & $204 / 324(63)$ & $389 / 741(52)$ & $261 / 665$ (39) & $854 / 1730(49)$ \\
\hline Systolic blood pressure, mean (SD) & $136.1(26.4)$ & $144.7(28.0)$ & $144.4(26.0)$ & $143.1(27.1)$ \\
\hline$\geq 140 \mathrm{~mm} \mathrm{Hg}$ & $162 / 385(42)$ & $541 / 973(56)$ & $516 / 924(56)$ & $1219 / 2282(53)$ \\
\hline Current smoker & $125(32)$ & $174(18)$ & $134(14)$ & $433(19)$ \\
\hline Revascularisation during admission & $295(76)$ & $414(42)$ & $175(19)$ & $884(38)$ \\
\hline GRACE risk score & $138(31)$ & $135(31)$ & $116(28)$ & $128(31)$ \\
\hline \multicolumn{5}{|c|}{ Documented preventive care received during index admission* } \\
\hline Smoking cessation advice/current smokers & $103 / 125(82)$ & $118 / 174(68)$ & $80 / 134(60)$ & $301 / 433(70)$ \\
\hline Screening for depression & $50(13)$ & $115(12)$ & $63(7)$ & $228(10)$ \\
\hline Dietary modification advice & $208(53)$ & $408(42)$ & $215(23)$ & $831(36)$ \\
\hline Physical activity advice & $247(63)$ & $491(50)$ & $250(27)$ & $988(43)$ \\
\hline Inpatient cardiac rehabilitation & $235(60)$ & $470(48)$ & $257(28)$ & $962(43)$ \\
\hline Seen by Indigenous health worker/Indigenous $\ddagger$ & $13 / 22(59)$ & $27 / 51(53)$ & $26 / 49(53)$ & $66 / 122(54)$ \\
\hline Referral to outpatient cardiac rehabilitation & $277(71)$ & $525(54)$ & $263(28)$ & 1065 (46) \\
\hline \multicolumn{5}{|l|}{ Medications prescribed at discharge* } \\
\hline Aspirin & $376(96)$ & $860(88)$ & $770(83)$ & $2006(87)$ \\
\hline Other antiplatelet & $335(86)$ & $696(71)$ & $420(45)$ & $1451(63)$ \\
\hline Statin/other lipid lowering therapy & 361 (93) & $843(86)$ & $740(80)$ & $1944(85)$ \\
\hline$\beta$-blocker & $319(82)$ & $749(76)$ & $572(62)$ & $1640(71)$ \\
\hline ACEi or A2RB & $296(76)$ & $649(66)$ & $561(60)$ & $1506(65)$ \\
\hline Diabetic drug§ & $62(17)$ & $242(27)$ & $231(28)$ & $535(23)$ \\
\hline Antidepressant & $43(12)$ & $124(14)$ & $177(22)$ & $344(15)$ \\
\hline
\end{tabular}

with hospital admissions databases during the study period. A total of 83 patients from the original cohort died in-hospital, and a further 2016 had a discharge diagnosis of non-ischaemic chest pain and are not included in this analysis of exposure to preventive care. The risk profile of the ACS cohort $(n=2299)$ was high (mean GRACE score 128 \pm 31 ). As expected, the GRACE risk score was higher in those with STEMI $(138 \pm 31)$ and NSTEMI $(135 \pm 31)$ than in those with UA $(116 \pm 28)$.

\section{Patient characteristics and preventive care at discharge}

Table 1 summarises the characteristics and clinical details for surviving patients with a diagnosis of ACS $(n=2299)$. Of those who experienced an ACS event (STEMI, NSTEMI, UA) $(\mathrm{n}=2299)$, two-thirds were male, $53 \%$ had a total cholesterol value $\geq 4.5 \mathrm{mmol} / \mathrm{L}, 49 \%$ had a blood glucose value $\geq 6.5 \mathrm{mmol} / \mathrm{L}, 53 \%$ had a systolic blood pressure $\geq 140 \mathrm{~mm} \mathrm{Hg}$, and $19 \%$ were documented as being current smokers. In terms of inpatient preventive care, approximately two-thirds of smokers received quit advice, only $10 \%$ were screened for depression, $36 \%$ received inpatient dietary advice, and $43 \%$ received physical activity advice. Among Indigenous ACS patients, only 54\% (66/122) were seen by an Indigenous Health Worker during their admission.

At discharge, $46 \%$ of all ACS patients were referred to an outpatient cardiac rehabilitation programme. Documented 
prescription rates of guideline recommended medicines for ACS (lipid lowering, aspirin, antiplatelet, $\beta$-blocker, ACEi/ARB) at discharge were $4-5(1490 / 2299,65 \%)$ or $2-3(632 / 2299$, $27 \%$ ), and $4 \%$ of patients were documented as having not been prescribed any pharmacotherapy. Of the patients with UA, 32\% $(299 / 928)$ had a GRACE risk score $\geq 130,68 \%$ (629/928) had a GRACE risk score <130, and 50\% (468/928) had experienced prior MI or had undergone PCI or CABG.

\section{Exposure to optimal preventive care}

Table 2 summarises the proportion of ACS patients who received optimal preventive care during their admission. In total, only $27 \%$ of patients received at least four medications (or two in the case of those with UA and a GRACE risk score $<130$ ), dietary or physical activity advice, and referral to cardiac rehabilitation. Rates of optimal care were highest among STEMI patients (51\%) and significantly lower for NSTEMI patients $(30 \%, \mathrm{p}<0.001)$ and patients with UA $(15 \%$, $\mathrm{p}<0.001)$. When additional emphasis was given to medication prescription (50\% weighting for medicines and 50\% for lifestyle advice and rehabilitation), 45\% (1041/2299) of the cohort were exposed to optimal care (medication prescription and lifestyle advice or rehabilitation referral).

\section{Factors associated with exposure to optimal preventive care}

The adjusted ORs and CIs describing the likelihood of receiving the various components of preventive care at the time of discharge are presented in figure 1. Factors associated with greater likelihood of being discharged with appropriate medications (at least four secondary prevention medications except in the case of UA patients with GRACE risk <130 where lipid lowering and aspirin alone were required) were: having a diagnosis of STEMI $(p=0.001)$ or NSTEMI $(p=0.021)$, undergoing PCI $(p<0.001)$ during the index admission, having had PCI or CABG before the index admission $(p<0.001)$, and having a history of prior hypertension $(\mathrm{p}=0.005)$. Factors associated with greater likelihood of referral to cardiac rehabilitation were: having a diagnosis of STEMI $(\mathrm{p}<0.001)$ or NSTEMI $(\mathrm{p}<0.001)$, having PCI $(\mathrm{p}<0.001)$ or CABG $(\mathrm{p}<0.001)$ during the index admission, or having a history of hypertension $(p=0.035)$. Being of older age $(p<0.001)$ and being admitted to a private hospital $(p=0.004)$ were associated with less likelihood of referral to rehabilitation. Factors associated with greater likelihood of provision of inpatient lifestyle advice while in-hospital were: diagnosis of STEMI $(\mathrm{p}<0.001)$ or NSTEMI $(\mathrm{p}<0.001)$, having PCI $(\mathrm{p}<0.001)$ or CABG $(\mathrm{p}<0.006)$ during the index admission, being obese $(p=0.042)$, and having a shorter length of stay $(p<0.001)$. Being older than 70 years $(p=0.012)$ was associated with lower likelihood of receiving inpatient lifestyle advice.

The adjusted ORs and CIs describing the likelihood of receiving optimal preventive care during the admission are presented in figure 2. Patients with a diagnosis of STEMI $(\mathrm{p}<0.0001)$ or NSTEMI $(p<0.0001)$, who had PCI $(p<0.0001)$ or CABG $(\mathrm{p}=0.011)$ during the index admission, or who had a history of hypertension before the index admission $(p=0.017)$ were more likely to receive optimal care. Patients aged over 70 years $(\mathrm{p}=0.002)$ and those admitted to a private hospital $(\mathrm{p}=0.003)$ were less likely to receive optimal care.

\section{DISCUSSION}

This cohort study provides a unique and comprehensive 'snapshot' of the preventive care delivered to 2299 patients admitted to Australian and New Zealand hospitals (public and private) with confirmed ACS at discharge. Despite universal guideline recommendations, around three-quarters of all patients admitted with ACS did not receive basic preventive care comprising proven pharmacotherapy, lifestyle advice, and referral to rehabilitation. Importantly, we classified receipt of lifestyle advice as any documented evidence of receipt of any form of dietary or physical activity advice, which is a very low bar in terms of individualised advice, and therefore it is highly likely that even fewer patients received individualised advice from appropriate health professionals. Further, the rate was still less than half when greater weighting was given to medication prescription. We also found that older age, diagnosis of UA, not having PCI, and being admitted to a private hospital were associated with lower likelihood of receiving optimal care. Therefore, this study not only highlights the clear variation in provision of preventive care across the ACS diagnosis spectrum, but also provides more detailed information about where opportunities exist to improve delivery of care.

International guidelines recommend that all patients with ACS should be referred to cardiac rehabilitation (CR), provided with basic lifestyle advice, and prescribed preventive pharmacotherapy. ${ }^{3}$ 6-8 In this study, only one-quarter of the cohort received this level of preventive care. In addition, Australian and other international guidelines recommend all patients should be screened for depression. ${ }^{3}$ We found that this only occurred in $10 \%$ of the ACS population. Further, only about half of the patients of Indigenous background were reviewed by an Indigenous health-worker during their hospital stay. This study found that $46 \%$ of ACS patients were referred to cardiac rehabilitation, but a 2010 report $(n=1545)$ by the National Prescribing Service of Australia found that even when patients were referred to cardiac rehabilitation, only half completed the programme. ${ }^{21}$ These findings are concerning and call for urgent

Table 2 Exposure to preventive care during ACS admission

\begin{tabular}{|c|c|c|c|c|}
\hline Guideline recommendation* & STEMI & NSTEMI & UA & All ACS \\
\hline Discharged on secondary prevention medicationst & $330 / 390(85)$ & $697 / 981(71)$ & $603 / 928(65)$ & $1630 / 2299(71)$ \\
\hline Inpatient advice about smoking cessation if a smoker & $103 / 125(82)$ & $118 / 174(68)$ & $80 / 134(60)$ & $301 / 433(69)$ \\
\hline At least 1 of diet or physical activity advice received & $259 / 390(66)$ & $521 / 981(53)$ & $279 / 928(30)$ & $1059 / 2299(46)$ \\
\hline Received referral to outpatient cardiac rehabilitation & $277 / 390(71)$ & $525 / 981(53)$ & 263/928 (28) & $1065 / 2299(46)$ \\
\hline Total (medicines+lifestyle advice+referral to cardiac rehabilitation) & $197 / 390(51)$ & 296/981 (30) & 135/928 (15) & $628 / 2299(27)$ \\
\hline
\end{tabular}

${ }^{*} n(\%)$, unless otherwise specified.

†Based on any four of the following-aspirin, other antiplatelet, statin or lipid-lowering agent, $\beta$-blocker or angiotensin II receptor blocker/ACE inhibitor except in the case of patients with UA and GRACE risk score $<130$ where at least prescription of aspirin and lipid lowering was required.

ACS, acute coronary syndrome; GRACE, Global Registry of Acute Coronary Events; NSTEMI, non-ST segment elevation myocardial infarction; STEMI, ST segment elevation myocardial

infarction; UA, unstable angina. 
Odds Ratio (95\% Cl)

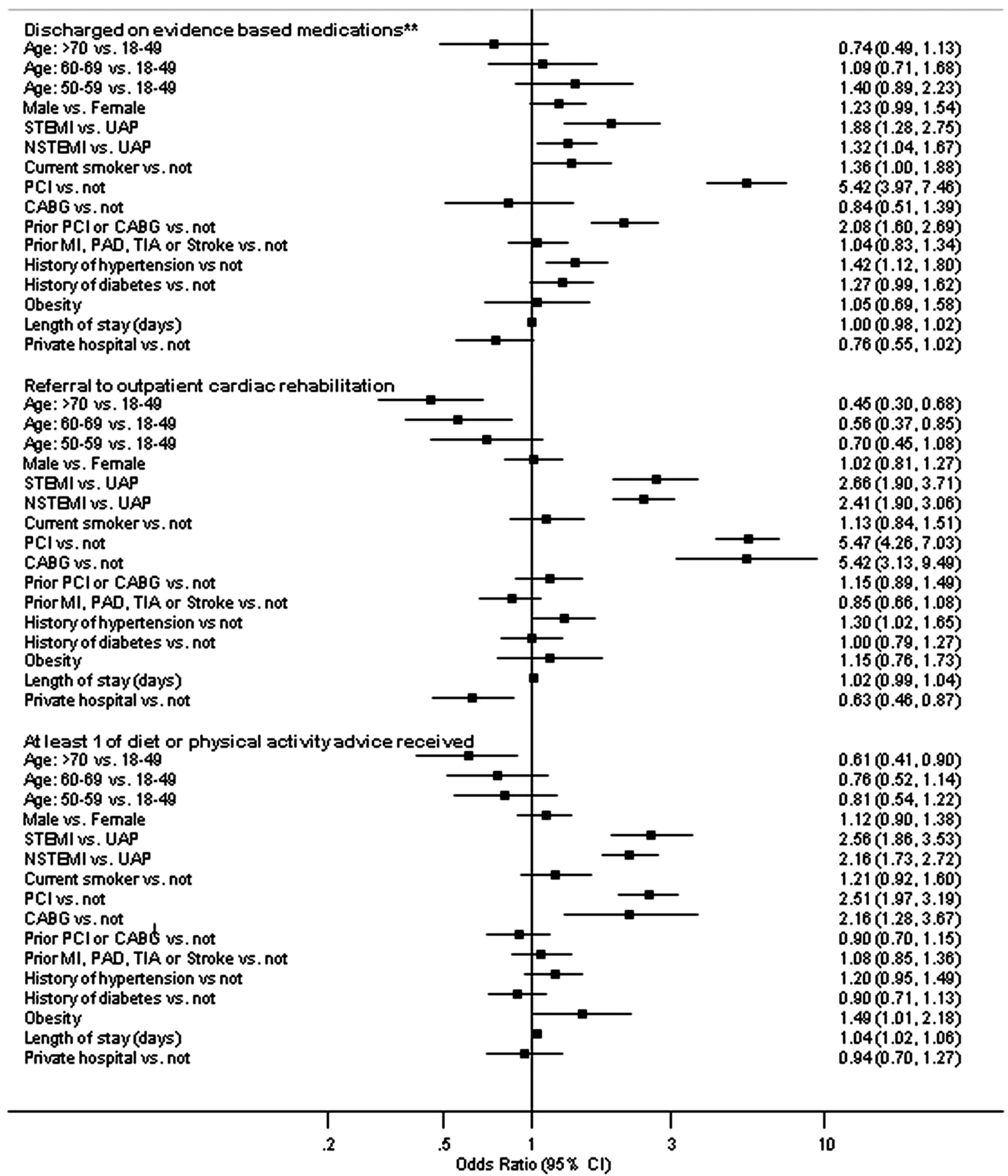

Figure 1 Adjusted ORs and $95 \% \mathrm{Cls}^{*}$ for likelihood of receiving at least four secondary prevention medicines on discharge, referral to cardiac rehabilitation, and inpatient lifestyle advice (OR $>1$ means likely). ${ }^{*}$ Using multilevel multivariable logistic regression. ${ }^{*}$ Based on any four of the following-aspirin, other antiplatelet, statin or lipid lowering agent, $\beta$-blocker or angiotensin II receptor blocker/ACE inhibitor except in the case of patients with unstable angina and GRACE risk score $<130$ where at least prescription of aspirin and lipid lowering was required. CABG, coronary artery bypass graft surgery; GRACE, Global Registry of Acute Coronary Events; MI, myocardial infarction; NSTEMI, non-ST segment elevation myocardial infarction; PAD, peripheral arterial disease; $\mathrm{PCl}$, percutaneous coronary intervention; STEMI, ST segment elevation myocardial infarction; TIA, transient ischaemic attack; UAP, unstable angina pectoris.

review and consideration of preventive care for patients with ACS bi-nationally. Optimising patient outcomes after ACS through standardisation of care has now emerged as a major near-term goal in the health agenda of Australia and New Zealand. ${ }^{22}$ This present study highlights the importance of inclusion of preventive care aspects within that agenda.

Our results are similar to other international studies. Two large prospective surveys of ACS patients across numerous
European countries have shown similar rates of pharmacotherapy to the Australian and New Zealand SNAPSHOT Registry. ${ }^{10}$ In the more recent European audit $(n=6385)$ compared to the bi-national SNAPSHOT, rates of aspirin were similar for STEMI (97\% vs 96\%) and NSTEMI (94\% vs 88\%). Corresponding results for statins were STEMI (81\% vs 93\%) and NSTEMI (74\% vs $86 \%$ ), and for ACEi/ARB were STEMI (75\% vs $76 \%$ ) and NSTEMI $(69 \%$ vs $66 \%) .{ }^{10}$ Our results for 
Odds Ratio $(95 \% \mathrm{Cl})$

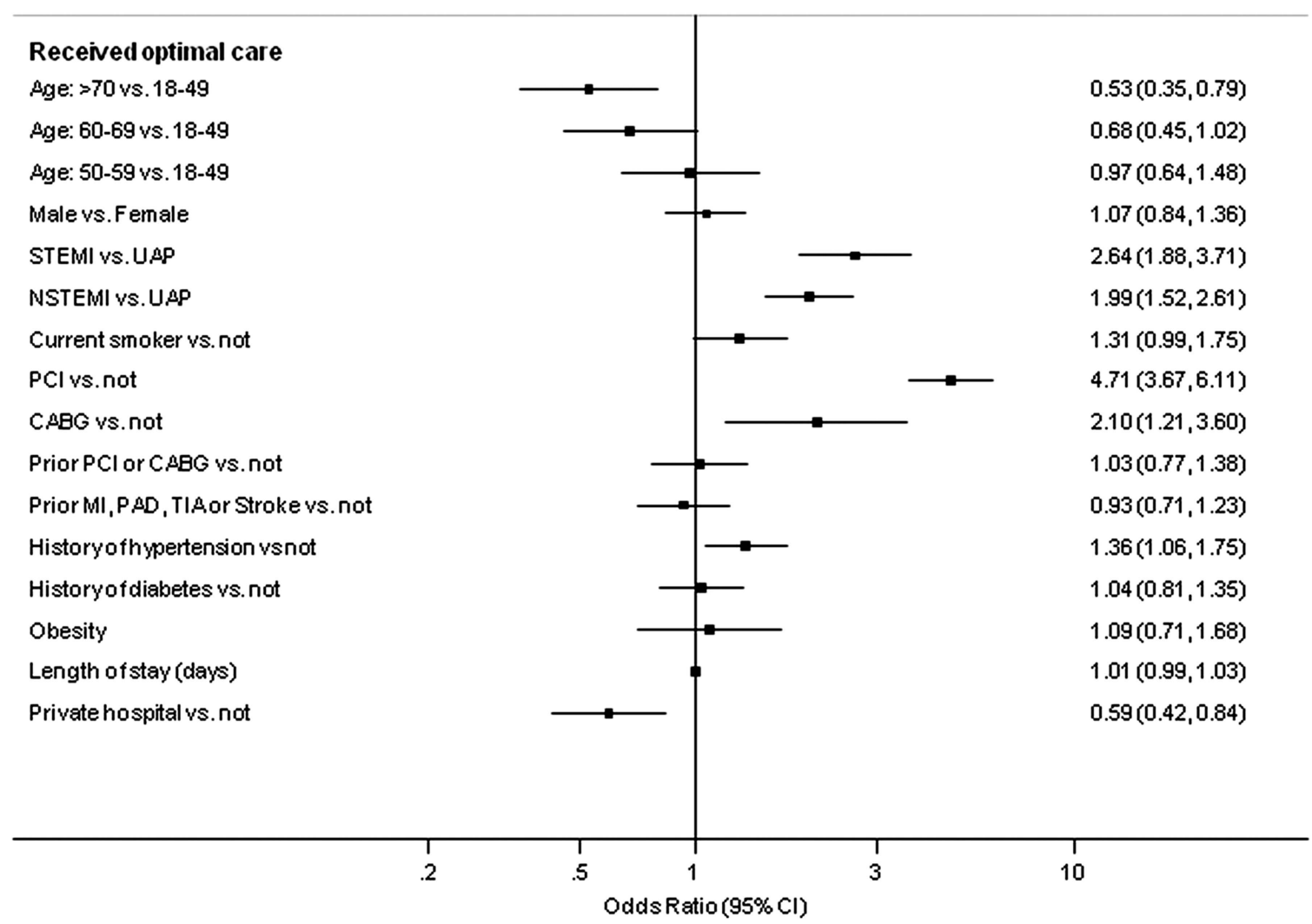

Figure 2 Adjusted ORs and $95 \% \mathrm{Cls}^{*}$ for likelihood of provision of optimal preventive care (OR $>1$ means more likely). *Using multilevel multivariable logistic regression. CABG, coronary artery bypass graft surgery; MI, myocardial infarction; NSTEMI, non-ST segment elevation myocardial infarction; PAD, peripheral arterial disease; PCI, percutaneous coronary intervention; STEMI, ST segment elevation myocardial infarction; TIA, transient ischaemic attack; UAP, unstable angina pectoris.

pharmacotherapy are also very similar to those reported by the American CRUSADE $(\mathrm{n}=64$ 426) national quality improvement initiative. ${ }^{23}$ Recent results of the Myocardial Ischaemia National Audit Project (MINAP) based in the UK report medication prescription of over 95\%, which is clearly higher than our Australian/New Zealand results; however, the MINAP cohort excludes patients who have been transferred, who have contraindications or who choose not to take medicines. ${ }^{24}$ In New Zealand, two previous ACS audits have also been crucial in defining treatment and resource gaps in that country over recent years. ${ }^{13} 14$ In the 2002 New Zealand audit of 695 discharged (alive) ACS patients and the $2007 \mathrm{New}$ Zealand audit of 815 discharged ACS patients, the use of discharge medications was also generally lower than in the present study including aspirin (82\% and $82 \%$, vs $87 \%$ ), $\beta$-adrenergic blockers $(63 \%$ and $66 \%$, vs $71 \%$ ), and statins (55\% and $70 \%$, vs $85 \%$ ). These New Zealand audits are now 5 and 10 years old and improvements in prescription of discharge pharmacotherapy, especially statin therapy, may have occurred. While our study shows similar rates of pharmacotherapy, very few of the previous audits have reported inpatient preventive lifestyle care and referral to rehabilitation in the same cohort.

Clinical diagnosis and PCI during the index admission were consistently associated with improved preventive care. Patients diagnosed with MI (STEMI or NSTEMI) were significantly more likely to receive secondary prevention pharmacotherapy, referral to rehabilitation, and inpatient lifestyle advice. Interestingly, older patients and those admitted to private hospitals were less likely to receive optimal preventive care. These differences could potentially be explained by shorter mean length of stay and potentially poorer documentation for patients admitted to the private hospitals, although this possibility requires further investigation. Unsurprisingly, those who had a longer hospital stay were more likely to receive inpatient dietary and physical activity advice, and those with obesity were more likely to receive inpatient dietary advice. This variability, particularly in relation to diagnosis, highlights the need for a more considered approach to the prevention of disease recurrence. Encouragingly, we confirmed that those with prior hypertension, prior vascular disease and PCI were more likely to receive secondary prevention pharmacotherapy.

The results of this study highlight extensive inequality in terms of the delivery of preventive care at a most vital time-the hospital admission. In particular diagnosis, intervention, age, and hospital type all impacted on the preventive care that patients received. Understanding why these inequities occur may help improve our health systems. The observations suggest that the practice patterns may reflect 'value judgements' where there may be a greater appreciation of value in the younger and STEMI patients. Documented risk stratification and discharge care planning may help overcome the perceived 'judgement' approach to provision of care. In addition, the development of standardised performance measures (for implementation in private and public hospitals) relating to secondary prevention are likely to be critical in providing objective evidence that optimal preventive care was provided. Such clinical standards and performance measures would need to take into account the diagnostic and therapeutic complexities for each patient. ${ }^{15}$ 
The strengths of this study are the inclusive nature of its design. This study is unique in its ability to provide insights into the provision of care but it is limited by the nature of data collection, namely, via analysis of the medical records. The design allows widespread and valuable benchmarking at a single time point rather than local continuous quality improvement to monitor change over time. This study highlights that valuable health system and public health insights can be obtained from a 'snapshot in time' at potentially much lower costs than large scale and ongoing audits. Further, although we have defined optimal care as having received inpatient lifestyle advice, medications and referral to rehabilitation on discharge, we are unable to determine the specific relative contributions of each of these aspects of care; future research is needed to investigate the ratio that each of these interventions may play in terms of future hospital readmissions and mortality.

This study is not without limitations. Most importantly, this was an audit project (without clinical outcomes) and reports data collected from medical records. As with any observational dataset, results need to be considered in the context of potential variation in record keeping between and within centres and issues of attribution, given that local researchers collected data within their area of employment. Also, data were collected based on hospital admissions during a 2-week period; this may not be representative of admissions over the entire calendar year and it may not be reflective of actions (eg, completion of rehabilitation) actually taken by patients after discharge. In addition, when analysing prescription of pharmacotherapy (despite the case report form allowing for removal of cases where medications were contraindicated or where there was drug intolerance/resistance) in a study such as this, it is difficult to account for the entirety of heterogeneity that exists between patients, particularly in those with UA. Although we have used the GRACE risk score as an indicator of risk, results should be

Key messages

What is already known about this subject?

- Approximately half of acute coronary events occur in individuals with prior disease.

- Internationally, acute coronary syndrome (ACS) guidelines recommend strategies targeting preventive care and highlight the importance of commencing preventive care immediately after ACS.

What does this study add?

- Provision of preventive care in patients admitted to hospital with ACS is suboptimal.

- Proportionally more ST segment elevation myocardial infarction (STEMI) patients, and those undergoing percutaneous coronary intervention, receive optimal preventive care.

- Older patients (>70 years) and those treated in private hospitals were less likely to receive optimal care.

\section{How might this impact on clinical practice?}

- Greater focus on in-hospital delivery of preventive care is needed to provide the essential foundation for lifelong secondary prevention.

- Improved provision of care to a broader range of patients is needed to ensure equity and access to preventive care during the inpatient admission. considered with this in mind. Finally, the study reports the preventive care received during the index admission, and data pertaining to what happens after the patients leave hospital is an area that requires ongoing research.

\section{CONCLUSIONS}

Only one-quarter of all patients admitted with ACS received optimal secondary prevention (pharmacotherapy, lifestyle advice, and referral to rehabilitation). This study provides unique insights into the provision of preventive therapy and lifestyle modification advice (or lack thereof) to patients admitted to hospital with an ACS. Proportionately more STEMI than non-STEMI and UA patients received guideline recommended preventive care. Findings also suggest that younger patients, those who have a PCI during admission, and those admitted to public hospitals are more likely to receive optimal preventive care. These findings highlight the persistence of the evidence treatment gaps in a contemporary cohort of ACS patients. Standardising inpatient care in line with guidelines is likely to contribute to more effective secondary prevention post-discharge.

\section{Author affiliations}

${ }^{1}$ The George Institute for Global Health, Sydney, Australia

${ }^{2}$ Sydney Medical School, University of Sydney, Sydney, Australia

${ }^{3}$ Department of Cardiovascular Medicine, Flinders University, Southern Adelaide Local Health Network, Adelaide, Australia

${ }^{4}$ Statewide Cardiac Clinical Network, South Australian Health; Flinders University, Adelaide, Australia

${ }^{5}$ Westmead Hospital, Sydney, Australia

${ }^{6}$ Cardiology Department, Concord Hospital, Sydney, Australia

${ }^{7}$ Queensland Health, Brisbane, Australia

${ }^{8}$ Cardiac Network, Agency for Clinical Innovation, Sydney, Australia

${ }^{9}$ National Heart Foundation of Australia (New South Wales Division) Sydney, Australia

${ }^{10}$ Royal Brisbane Hospital, Brisbane, Australia

${ }^{11}$ Green Lane CVS Service, Auckland City Hospital, Auckland, New Zealand

${ }^{12}$ Liverpool Hospital Sydney, Australia

${ }^{13}$ University of New South Wales, Sydney Australia

${ }^{14}$ School of Population Health, University of Western Australia, Perth, Australia

Acknowledgements Steering Committee: David Brieger (NSW), Co-chair, John French (NSW) Co-chair, Derek Chew (SA) Co-chair, Chris Ellis (NZ), Co-Chair, Gerry Devlin (NZ), Co-Chair, Chris Hammett (QLD), Bernadette Aliprandi-Costa (NSW), Isuru Ranasinghe (NSW), Bridie Carr (NSW), Julie Redfern (NSW), Fiona Turnbull (NSW), Carolyn Astley (SA), Tom Briffa (WA), Jamie Rankin (WA), Ahmad Farshid (ACT), Pearl Taverner (ACT), Darren Walters (QLD), Stephen Bloomer (WA), John Elliott (NZ), Jeff Lefkovits (Vic), Karice Hyun (NSW). Project managers- State-based Cardiac Clinical networks. New Zealand- Greg Gamble, NSW/ACT- Bernadette Aliprandi-Costa, Bridie Carr, Karen Lintern, Pearl Taverner, Queensland- Tegwen Howell, Cindy Hall, Susanne Spencer, Dayna Williamson, Victoria- Hella Parker, Julie Plunkett, Wendy

Wallace-Mitchell, South Australia, TAS, NT- Rosanna Tavella, Carolyn Astley, Western Australia- Samantha Thompson. Data Management and analysis- The George Institute for Global Health and the South Australian Health and Medical Research Institute (SAHMRI): Associate Professor Fiona Turnbull, Dr Isuru Ranasinghe, Associate Professor Julie Redfern, Karice Hyun, Matthew Horsfall, Helen Hughes.

Contributors All authors have been involved in the study from inception and have approved the final manuscript.

Funding The SNAPSHOT ACS study was supported in part by: The Cardiac Society of Australia and New Zealand; The National Heart Foundation of Australia, The Agency for Clinical Innovation (NSW), the Victorian Cardiac Clinical Network, the Queensland Cardiac Clinical Network, the Cardiovascular Health Network, Department of Health, WA and the State-wide Cardiac Clinical Network, South Australian Health. The study was endorsed by the Australian Commission for Quality and Safety in Health Care and supported with in-kind support from each of the participating hospitals and their respective State and Territory Departments of Health. JR is funded by a NHMRC Career Development Fellowship (APP1061793) co-funded with a National Heart Foundation Future Fellowship (G160523). CC is funded by a Career Development Fellowship co-funded by the NHMRC and National Heart Foundation of Australia (1033478) and Sydney Medical Foundation Chapman Fellowship. FT is supported by a National Heart Foundation of Australia Career Development Award. 
Competing interests DC: lecture fees AstraZeneca Australia; educational programme: Heart.org. JF: Advisory Board Membership Sanofi Aventis Australia, AstraZeneca Australia. Eli Lilly Australia and Boehringer Ingelheim; Grant In Aid; The Medicines Company. TB: Grant In Aid WA Department of Health; travel support WA Department of Health. CH: Consultancy Bayer Australia and Eli Lilly Australia; lecture fees Boehringer Ingelheim and Eli Lilly Australia; travel assistance AstraZeneca Australia, Bayer Australia, Boehringer Ingelheim Australia and Eli Lilly Australia, Schering Plough Australia, Abbott Medical Australia. TH: travel assistance Heart Foundation Australia. DB: Advisory Board AstraZeneca Australia, Boehringer Ingelheim Australia, Bayer Australia, Pfizer, BMS Australia; Grants: AstraZeneca Australia, Sanofi Aventis Australia, Merck Schering Plough Australia, Boehringer Ingelheim Australia; lecture fees AstraZeneca Australia, Bayer Australia; travel assistance Bayer Australia, Boehringer Ingelheim Australia.

Ethics approval NSW Cancer Institute Human Research Ethics Committee (Population Division).

Provenance and peer review Not commissioned; externally peer reviewed.

Open Access This is an Open Access article distributed in accordance with the Creative Commons Attribution Non Commercial (CC BY-NC 3.0) license, which permits others to distribute, remix, adapt, build upon this work non-commercially, and license their derivative works on different terms, provided the original work is properly cited and the use is non-commercial. See: http://creativecommons.org/ licenses/by-nc/3.0/

\section{REFERENCES}

1 World Health Organisation: The top 10 causes of death. 2004 October 2008; Fact sheet N³10. http://www.who.int/mediacentre/factsheets/fs310/en/index.html

2 Australian Institute of Health and Welfare (AlHW). Impact of falling cardiovascular disease death rates: deaths delayed and years of life extended. Bulletin no. 70. Cat. no. AUS 113. Canberra: AlHW, 2009.

3 Aroney CN, Aylward P, Kelly AM, et al.; for the Acute Coronary Syndrome Guidelines Working Group. Guidelines for the management of acute coronary syndromes 2006. Med J Aust 2006;184:51-30.

4 Chew D, Amerena J, Coverdale S, et al. Invasive management and late clinical outcomes in contemporary Australian management of ACS: observations from the ACACIA registry. Med J Aust 2008;188:691-7.

5 Briffa T, Hobbs M, Tonkin A, et al. Population trends of current coronary heart disease event rates remain high. Circ Cardiovasc Qual Outcomes 2011:4:107-13.

6 Steg PG, James SK, Atar D, et al. ESC guidelines for the management of acute myocardial infarction in patients presenting with ST segment elevation: the task force on the management of ST-segment elevation acute myocardial infarction of the European Society of Cardiology (ESC). Eur Heart J 2012:33:2569-619.

7 Bassand JP, Hamm CW, Ardissino D, et al. Guidelines for the diagnosis and treatment of non-ST-segment elevation acute coronary syndromes The Task Force for the diagnosis and treatment of non-ST-segment elevation acute coronary syndromes of the European Society of Cardiology. Eur Heart J 2007;28:1598-660.

8 Anderson JL, Adams CD, Antman EM, et al. 2011 ACCF/AHA focused update incorporated into the ACC/AHA 2007 guidelines for the management of patients with unstable angina/non-ST-elevation myocardial infarction: a report of the
American College of Cardiology Foundation/American Heart Association Task Force on Practice Guidelines. Circulation 2011;123:e426-579.

9 Carruthers KF, Dabbous OH, Flather MD, et al:; for the GRACE Investigators. Contemporary management of acute coronary syndromes: does the practice match the evidence? The Global Registry of Acute Coronary Events (GRACE). Heart 2005;91:290-8.

10 Mandelzweig L, Battler A, Boyko V, et al. The second Euro Heart Survey on acute coronary syndromes: characteristics, treatment, and outcome of patients with ACS in Europe and the Mediterranean Basin in 2004. Eur Heart J 2006;27:2285-93.

11 Chew DP, Amerena JV, Coverdale SG, et al. Invasive management and late clinical outcomes in contemporary Australian management of acute coronary syndromes: observations from the ACACIA registry. Med J Aust 2008;188:691-7.

12 Hasdai D, Behar S, Wallentin L, et al. A prospective survey of the characteristics, treatments and outcomes of patients with acute coronary syndromes in Europe and the Mediterranean basin: the Euro Heart Survey on Acute Coronary Syndromes (Euro Heart Survey ACS). Eur Heart J 2002;23:1190-201.

13 Ellis C, Devlin G, Matsis P, et al. Acute coronary syndrome patients in New Zealand receive less invasive management when admitted to hospitals without invasive facilities. N Z Med J 2004;117:U954.

14 Ellis C, Gamble G, Hamer A, et al. Patients admitted with an acute coronary syndrome (ACS) in New Zealand in 2007: results of a second comprehensive nationwide audit and a comparison with the first audit from 2002. NZ Med J 2010;123:25-43.

15 Chew DP, French J, Briffa T, et al. The status of acute coronary syndrome care across Australia and New Zealand: primary observations from the SNAPSHOT ACS study. Med J Aust 2013;199:185-91.

16 Gislason GH, Rasmussen JN, Abildstrøm SZ, et al. Long-term compliance with beta-blockers, angiotensin-converting enzyme inhibitors, and statins after acute myocardial infarction. Eur Heart J 2006;27:1153-8.

17 Pack QR, Mansour M, Barboza JS, et al. An early appointment to outpatient cardiac rehabilitation at hospital discharge improves attendance at orientation: a randomized, single-blind, controlled trial. Circulation 2013;127:349-55.

18 Granger $\mathrm{CB}$, Goldberg RJ, Dabbous $\mathrm{OH}$, et al.; for the Global Registry of Acute Coronary Events Investigators. Predictors of hospital mortality in the global registry of acute coronary events. Arch Intern Med 2003;163:2345-53.

19 Unal B, Critchley JA, Capewell S. Explaining the decline in coronary heart disease mortality in England and Wales between 1981 and 2000. Circulation 2004;109:1101-7.

20 Ford ES, Ajani UA, Croft JB, et al. Explaining the decrease in U.S. deaths from coronary disease, 1980-2000. N Engl J Med 2007;356:2388-98.

21 National Prescribing Service. Multicentre drug use evaluation in hospitals discharge

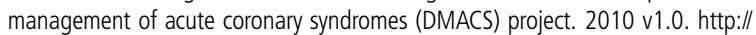
www.nps.org.au/_data/assets/pdf_file/0014/114107/damcs_report_dec2010.pdf (accessed 14 Jun 2013).

22 Smith MC. From the Australian Commission on Safety and Quality in Health Care. Towards national clinical care standards. Med I Aust 2012;197:540.

23 Ohman EM, Roe MT, Smith SC, et al. Care of non-ST-segment elevation patients: insights from the CRUSADE national quality improvement initiative. Am Heart $J$ 2004;148:S34-9.

24 Myocardial Ischaemia National Audit Project. How the NHS cares for patients with heart attack. Annual Public Report April 2012-March 2013. University College London. 